\title{
A retrospective study on the clinico-demographic profile of multiple sclerosis patients in various tertiary care hospitals in Mangalore city of South India
}

\author{
Nitin Joseph ${ }^{1}$, Rachel Melissa Salins², Priyanka Ramesh², Narayana Venkatesh Krishna ${ }^{3}$ \\ ${ }^{1}$ Department of Community Medicine, Kasturba Medical College, Mangalore, Manipal Academy of Higher Education, \\ Manipal, India \\ ${ }^{2}$ Kasturba Medical College, Mangalore, Manipal Academy of Higher Education, Manipal, India \\ ${ }^{3}$ Department of Community Medicine, Father Muller Medical College, Mangalore, India
}

\begin{abstract}
Objectives. To study the risk factors, clinical presentations, course of the disease, investigations and management practices in MS.

Materials and methods. Records of 23 patients diagnosed with MS using the McDonald diagnostic criteria were analysed.

Outcomes. The mean age at diagnosis was $34.6 \pm 17.1$ years. 17 (73.9\%) patients were females. Early and late onset MS were present among $2(8.7 \%)$ and $4(17.4 \%)$ patients respectively. The most common symptom at diagnosis was paraesthesia [9 (39.1\%)]. Motor symptoms and sensory symptoms at the time of diagnosis were present among 9(39.1\%) patients each.

$20(87 \%)$ patients had motor system involvement, 9 (39.1\%) had sensory system involvement, 12 (52.2\%) had cranial nerve involvement, $4(17.4 \%)$ had sphincter dysfunction and $2(8.7 \%)$ had cerebellar involvement. Relapsing and remitting MS (RRMS) were present among 18 (78.3\%) patients. Oligoclonal bands were present among 17 (73.9\%) patients. Vitamin D levels in the blood were found deficient among $4(80 \%)$ out of 5 patients with available reports of the same. Juxtacortical region was the most common [9 (39.1\%)] site of involvement. Steroids were used for management among majority [12 (52.2\%)] of the patients. Non-pharmacological methods for management constituted only physiotherapy which was advised among 13 (56.5\%) patients. Improvement in treatment outcome were seen among 19 (82.6\%) patients.

Conclusions. Almost three fourth of the patients were females. RRMS was the most common course of the disease. Juxtacortical region was the most common site involved. Steroids were most commonly used for management. More non-pharmacological methods need to be introduced for MS management.
\end{abstract}

Keywords: multiple sclerosis, risk factors, clinical features, investigations, management, hospital based study

\section{INTRODUCTION}

Multiple sclerosis (MS) is a chronic inflammatory disease associated with demyelination and degeneration of the nervous system. It leads to both cognitive and physical disability among the affected. In the Western world, it has been reported to be the most common cause of non-traumatic neurological disability among young adults [1].

The total MS patients in India in 2016 was estimated to be 204,460 (uncertainty Interval 182,397 to 231,531) [2]. In 2013, the crude prevalence of MS in the current setting, namely Mangalore city, was 8.3 per 100,000 population [3]. 
Its occurrences and distributions are heterogeneous due to the differences in the genes and the environmental conditions at various settings [4]. Heterogeneity is also seen in the course of the disease and its response to treatment [5].

The current approach in MS management aims to prevent neurodegeneration by starting treatment early during the inflammatory stages of the disease [5]. Considering the various immunopathological subtypes of MS, clinical profiling of the patients help in undertaking appropriate management practices [6]. The detailed profiling exercise need to be complemented by assessment of biomarkers and various diagnostic modalities which help in the decision making process for providing individualized therapeutic care [7]. This study was therefore done to study the risk factors, clinical presentations, course of the disease, investigations and management practices in MS, among patients attending various tertiary care hospitals in Mangalore city of south India.

\section{MATERIALS AND METHODS}

This study was conducted at one government and three private tertiary care hospitals, affiliated to two private medical colleges during the months March, April and May 2019. It was a retrospective record based cross sectional study. The ethics approval was first obtained from Kasturba Medical College Institutional Ethics Committee on 20th March 2019. The approval number was IEC KMC MLR 03-19/82. It was then obtained from Father Muller Medical College Institutional Ethics Committee dated 14th May 2019. The approval number was FMMCIEC/CCM/129/2019.

Records of all confirmed patients with MS over the past 10 years (starting from 2009), at these hospitals, were analysed and the information were recorded in a proforma. Patients were diagnosed using the McDonald diagnostic criteria for MS. The proforma was content validated by a neurologist. Data regarding socio demographic details, age at diagnosis, clinical symptoms and signs, course of the disease, co-morbidities, risk factors like genetic defects, family history of MS, investigation findings, treatment and its outcomes were recorded by the investigators.

Data entry and analysis were done using IBM SPSS for Windows version 25.0, Armonk, New York. Fisher's Exact test was used to test association. $p$ value $<0.05$ was used as the criterion for significance.

\section{RESULTS}

23 patients with multiple sclerosis were included in this study.
The mean age of the patients was $36.6 \pm 16.8$ years and it ranged from 20 years to 74 years. Majority were females [17 (73.9\%)] and were from urban areas $[20(87 \%)]$ (Table 1$)$. The mean age at diagnosis was $34.6 \pm 17.1$ years. It ranged from 18 years to 73 years. The mean age at diagnosis among males was $45.7 \pm 19.5$ years and among females was $31.3 \pm 15.8$ years $(\mathrm{t}=1.318, \mathrm{P}=0.214)$. The proportion of patients with early onset or juvenile MS ( $\leq 18$ years) were $2(8.7 \%)$. Both these patients were females $(\mathrm{P}=$ 1). Late onset of disease ( $\geq 50$ years) were seen among $4(17.4 \%)$ patients. Among them, males and females were 2 patients each $(\mathrm{P}=0.2705)$.

There was no patient with positive history of genetic diseases or any other autoimmune diseases or with any family history of MS. Five (21.7\%) patients were vegetarians.

TABLE 1. Socio-demographic distribution of patients with multiple sclerosis

\begin{tabular}{|c|c|c|}
\hline Socio-demographic variables & Number & Percentage \\
\hline \multicolumn{3}{|l|}{ Age distribution (years) } \\
\hline 20 & 2 & 8.7 \\
\hline $21-30$ & 10 & 43.5 \\
\hline $31-40$ & 4 & 17.4 \\
\hline $41-50$ & 3 & 13.1 \\
\hline $51-60$ & 1 & 4.3 \\
\hline $61-70$ & 1 & 4.3 \\
\hline$>70$ & 2 & 8.7 \\
\hline \multicolumn{3}{|l|}{ Gender } \\
\hline Males & 6 & 26.1 \\
\hline Females & 17 & 73.9 \\
\hline \multicolumn{3}{|l|}{ Marital status } \\
\hline Married & 14 & 60.9 \\
\hline Unmarried & 9 & 39.1 \\
\hline \multicolumn{3}{|l|}{ Occupation $(n=4)$} \\
\hline Labourers & 2 & 50.0 \\
\hline Student & 1 & 25.0 \\
\hline Teacher & 1 & 25.0 \\
\hline \multicolumn{3}{|l|}{ Place of residence } \\
\hline Urban & 20 & 87.0 \\
\hline Rural & 3 & 13.0 \\
\hline Total & 23 & 100.0 \\
\hline
\end{tabular}

Out of the 23 patients, 14 (60.9\%) did not suffer from any co-morbidities. Twelve (52.2\%) among the MS patients were monosymptomatic and 11 (47.8\%) were polysymptomatic at the time of diagnosis. The most common symptoms at diagnosis were paraesthesia [9 (39.1\%)] followed by vision impairment [7 (30.4\%)]. Motor symptoms and sensory symptoms at the time of diagnosis were present among 9(39.1\%) patients each (Table 2). 
TABLE 2. Distribution of clinical features among patients with multiple sclerosis $(n=23)$

\begin{tabular}{|c|c|c|}
\hline Characteristics & Number & Percentage \\
\hline \multicolumn{3}{|l|}{ Symptoms at diagnosis* } \\
\hline Paraesthesia & 9 & 39.1 \\
\hline Vision impairment & 7 & 30.4 \\
\hline Difficulty in walking & 5 & 21.7 \\
\hline Bowel/bladder dysfunction & 4 & 17.4 \\
\hline Severe fatigue & 4 & 17.4 \\
\hline Eye pain & 2 & 8.7 \\
\hline Reduced cutaneous sensations & 2 & 8.7 \\
\hline Vertigo & 2 & 8.7 \\
\hline Headache & 2 & 8.7 \\
\hline Movement disorders & 2 & 8.7 \\
\hline Others ${ }^{\#}$ & 5 & 21.7 \\
\hline \multicolumn{3}{|l|}{ Signs at diagnosis* } \\
\hline Decreased muscle power & 13 & 56.5 \\
\hline Diminished visual acuity & 7 & 30.4 \\
\hline Extensor plantar reflex & 5 & 21.7 \\
\hline Absent tendon reflex & 4 & 17.4 \\
\hline Exaggerated tendon reflex & 3 & 13.0 \\
\hline Quadriplegia & 2 & 8.7 \\
\hline Hypertonia & 2 & 8.7 \\
\hline Ataxia & 2 & 8.7 \\
\hline Otherst & 2 & 8.7 \\
\hline \multicolumn{3}{|l|}{ Course of the disease } \\
\hline Primary progressive & 2 & 8.7 \\
\hline Clinically isolated syndrome & 2 & 8.7 \\
\hline Relapsing and remitting & 18 & 78.3 \\
\hline Chronic progressive & 1 & 4.3 \\
\hline \multicolumn{3}{|l|}{ Co-morbidities* } \\
\hline Thyroid disorders & 4 & 17.4 \\
\hline Hypertension & 2 & 8.7 \\
\hline Psychosis & 2 & 8.7 \\
\hline Migraine/Tension headache & 2 & 8.7 \\
\hline Ischaemic heart disease & 1 & 4.3 \\
\hline
\end{tabular}

*Multiple responses

"Difficulty in speech 1, Breathing difficulty 1, Behavioural changes 1, Back pain 1, Restriction of movement of knee joint 1 +Paraplegia 1, Hypotonia 1

One among the patients with early onset MS and 3 among the patients with late onset MS had motor symptoms at the time of presentation $(\mathrm{P}=1)$. None among the patients with early onset MS and 1 among the patients with late onset MS had sensory symptoms at the time of presentation $(\mathrm{P}=1)$. Out of the total 23 patients, 20 (87\%) had motor system involvement, 9 (39.1\%) had sensory system involvement, 12 (52.2\%) had cranial nerve involvement, 2 (8.7\%) had cerebellar involvement and 4 (17.4\%) had sphincter dysfunction. At the time of diagnosis, both the patients with early onset MS presented with visual impairment, while 1 each of them presented with movement disorders and difficulty in walking.

At the time of diagnosis, symptoms like vision impairment, vertigo and paraesthesia were present in 1 patient each and signs like bowel/bladder dysfunction and difficulty in speech were present among 1 out of the 4 patients with late onset MS.

Relapsing and remitting MS (RRMS) were present among 18 (78.3\%) patients. RRMS was seen among both the patients with early onset MS. It was also present among 3 out of 4 patients with late onset MS. One of the patient with late onset MS had clinically isolated syndrome (CIS). Neuromyelitis optica spectrum disorder (NMOSD) or Devic's disease was present in one patient.

Vitamin D levels in the blood were found deficient among $4(80 \%)$ out of 5 MS patients with available reports of the same. Oligoclonal bands (OCBs) in the cerebro spinal fluid (CSF) were found in 17(73.9\%) patients Juxtacortical region was the most common site of involvement among majority [9(39.1\%)] of the patients as identified in the MRI scan (Table 3).

TABLE 3. Laboratory and investigatory findings among patients with multiple sclerosis $(n=23)$

\begin{tabular}{|l|l|l|}
\hline Laboratory parameters & Number & Percentage \\
\hline Vitamin D levels $(\mathrm{n}=5)$ & & \\
\hline Deficient $(<20 \mathrm{ng} / \mathrm{ml})$ & 4 & 80.0 \\
\hline Normal & 1 & 20.0 \\
\hline ESR levels $(\mathrm{n}=12)$ & & \\
\hline Normal & 10 & 83.3 \\
\hline Raised & 2 & 16.7 \\
\hline Blood urea levels $(\mathrm{n}=14)$ & & \\
\hline Normal & 8 & 57.1 \\
\hline Raised & 6 & 42.9 \\
\hline Blood creatinine levels $(\mathrm{n}=14)$ & & \\
\hline Reduced & 1 & 7.1 \\
\hline Normal & 12 & 85.8 \\
\hline Raised & 1 & 7.1 \\
\hline MRI findings ${ }^{\#}$ & 23 & 100.0 \\
\hline CSF examination findings ${ }^{\dagger}$ & 23 & 100.0 \\
\hline Collagen profile $\ddagger$ & 6 & 26.1 \\
\hline Funduscopy§ & 5 & 21.7 \\
\hline $\begin{array}{l}\text { Electrophysiological studies of the } \\
\text { nerves }\end{array}$ & 5 & 21.7 \\
\hline CT scan findings** & & \\
\hline
\end{tabular}

"Lesions in: Juxtacortical region 9, Periventricular region 8, Cervical spine 5, Pyramidal tract 4, Cerebellum 3, Pons 3, Parietal lobe 3, Optic nerve 3, Optico brainstem cerebello spinal region 3, Brainstem 3, Thoracic spine 3, Calloso-septal interface 2, Corpus callosum 2, Frontal lobe 2, Juxtacallosal region 1, Insular region 1, Temporal lobe 1, Occipital lobe 1, Gangliocapsular region 1, External capsule 1, Midbrain 1, Dorsolumbar spine 1, Opticospinal region 1, Spinothalamic region 1, Acute transverse myelitis 1

+ Oligoclonal bands 17, Raised protein levels 11, Increase in $T$ Lymphocytes 11, Adenosine deaminase present 3, Raised gamma globulins 2, Raised IgG antibodies 1, Raised beta-1 Globulins 1, Increase in neutophils 1 , Normal CSF report 1

\# Normal 3, ANA raised 2, ANA borderline 1

$\S$ Optic atrophy 3, Macular edema 2

"Normal 2, Prolonged latency 2, Mild prolonged latency 1

**Bulging of the cervical spinal cord and indenting anterior subarachnoid space 1, Demyelination of spinal nerves 1 
Steroids like methylprednisolone and prednisolone were prescribed among 12 (52.2\%) patients. Immunosuppressant like azathioprine was prescribed among 10 (43.5\%) patients. Disease modifying drugs (DMDs) such as beta interferons and Teriflunomide were prescribed among 9 (39.1\%) patients (Table 4). All the patients on DMDs had RRMS. Non-pharmacological method constituted only physiotherapy and it was advised among 13 (56.5\%) patients. Improvement in treatment outcome were seen among 19 (82.6\%) patients. One out of the 2 patients with early onset MS $(\mathrm{P}=0.3241)$ and 2 out of the 4 patients with late onset MS ( $\mathrm{P}=$ 0.1246) showed improvement with treatment.

TABLE 4. Management practices among patients with multiple sclerosis $(n=23)$

\begin{tabular}{|l|l|l|}
\hline Management* & Number & Percentage \\
\hline Azathioprine & 10 & 43.5 \\
\hline Methylprednisolone & 9 & 39.1 \\
\hline Beta Interferons & 8 & 34.8 \\
\hline Prednisolone & 3 & 13.0 \\
\hline Vitamin D3 & 3 & 13.0 \\
\hline B complex vitamins & 2 & 8.7 \\
\hline Myoinositol & 2 & 8.7 \\
\hline Antioxidants & 2 & 8.7 \\
\hline Others\# & 8 & 34.8 \\
\hline Physiotherapy & 13 & 56.5 \\
\hline Treatment outcome & & \\
\hline Improved with treatment & 19 & 82.6 \\
\hline Did not improve & 4 & 17.4 \\
\hline
\end{tabular}

${ }^{*}$ Multiple responses

"Mycophenolate Mofetil 1, Teriflunomide 1, Baclofen 1, Gabapentin 1, Thiamine 1, Calcitriol 1, Multivitamins 1, Calcium 1

\section{DISCUSSION}

The mean age at diagnosis of MS in this study was 34.6 years. In studies done in Egypt [8], Chandigarh, India [9], Brazil [10] and New Delhi, India [11], the mean age at onset was 25.6, 28.5, 32.2 and 33.3 years respectively. The delayed age at diagnosis in this study as a consequence of delayed age at onset could be due to the influence of varying genetic and environmental factors in the setting on the disease aetiology. Previous Indian studies reported, mean age at onset among males as 31.8 years [12] and 33.5 years [13] and among females as 29.1 years [12] and 26.4 years [13]. Similar observation was made in the present study where the mean age at diagnosis was earlier among females. In this study, $8.7 \%$ of the total patients had early onset of MS. Early onset have been reported among 3 to 5\% MS patients [14]. Both the patients with early onset MS in this study and $77.5 \%$ in an Italian study were females [15]. The late age at onset of MS were reported among $17.4 \%$ patients in this study. This was more than the proportion of $6.3 \%$ reported in the Egyptian study [8]. Half of the patients with history of late onset MS in the present study, and $36.9 \%$ in a Canadian study were males [16].

In this study $73.9 \%$ and $60 \%$ to $90.7 \%$ of MS patients in previous studies were females [10,12,15,1721]. Like most other autoimmune disease, MS is also seen more commonly among females [22]. In a study done in Kashmir, India [20], most patients (90.2\%) were from rural areas which was in contrast to our findings where most patients were from urban areas. In another study done by Malli et al in Mangalore, urban living was a significant risk factor for MS [23]. These observations again support the role of local environmental factors in MS aetiology.

As many as $80 \%$ patients with known status of vitamin $\mathrm{D}$ in the present study, had deficient levels in the blood. A study done in Mangalore, by Pandit et al reported that vitamin D levels were significantly lower among MS patients [24]. In the present study, $21.7 \%$ patients were vegetarians. Malli et al. reported that vegetarian diet was a significant risk factor for MS [23]. There was no report of family history of MS among any of the patients in this study. However $4.6 \%$ of MS patients in a Sudanese study [21] and $13.8 \%$ in an Italian study [15] had positive history of the same.

Majority of the patients in this study and $81.1 \%$ patients in the Egyptian study were monosymptomatic at the time of diagnosis. [8] The most common symptoms at the time of diagnosis in this study were sensory paraesthesia followed by vision impairment and difficulty in walking. In the study done in Calcutta, India, visual impairment (33\%) followed by weakness of the limbs (31\%) and paraesthesia (20\%) were the most common initial symptoms in MS [12]. Another study done in Kashmir, India reported, visual involvement (40\%) followed by weakness of the limbs (35\%) and sphincter involvement (20\%) as the most common presenting symptoms [13]. Reduced visual acuity were reported among 27.6\% MS patients in the Sudanese study compared to $30.4 \%$ reported in this study [21]. Optic nerve involvement were reported among 57\% patients in the study done in Chandigarh, India [9] and among $3(13 \%)$ patients in this study. Visual involvement is a common feature in the Asian variety of MS [25]. Motor symptoms at the time of diagnosis were reported by $39.1 \%$ patients in this study and by $35.3 \%$ [21], 50\% [26] and 62.3\% [19] patients in studies done elsewhere. Sensory symptoms at the time of diagnosis were also reported by $39.1 \%$ patients in this study which was more than the proportion of $10 \%$ reported in the study done in Kashmir, India [13]. In this study, NMOSD was present in one patient. Its prevalence in south Indian population was found to be 2.7 per 100,000 population [3]. In this 
study, psychosis was present among $8.7 \%$ patients. Psychiatric problems such as depression (22.2\%), anxiety (21\%) and aggressive behaviour (13.7\%) were also reported in a Canadian study [18] In a study done in Bangalore, India, $51.6 \%$ of MS patients suffered from depression [27]. Severe fatigue was reported by $17.4 \%$ patients in this study. In the study done in Bangalore, India, depression was associated with fatigue among MS patients [27]. This emphasizes the need for screening various psychiatric disorders while managing patients with MS. In the Asian variety of MS, spinal cord and optic nerve involvement were seen commonly [25]. In a study done in Calcutta, India, lesions in the posterior column and spinothalamic regions were present in $55 \%$ and $51 \%$ patients respectively [12]. The study done in Kashmir, India reported, pyramidal tract (70\%) followed by spinothalamic and posterior column (50\%) and cerebellar (25\%) region as the most common site of lesion in MS [13]. Another study done in Chandigarh, India reported pyramidal tract involvement among $87 \%$ patients [9]. In the present study, lesions were more common in the juxtacortical region.

Majority of patients (78.3\%) in this study had RRMS. In about $85 \%$ patients, MS is known to begin as an acute clinical episode or CIS that evolves into a RRMS course. After a gap of about 15 years, it progresses into a secondary progressive MS (SPMS) course that leads to chronic disability. The other $15 \%$ patients start with the primary progressive MS (PPMS) course of the disease [28]. Previous studies reported CIS among 4.5\% [8], 13.3\% [29], 52.7\% [15], PPMS among $0 \%$ to $48.9 \%$ [8-13,20,29,30], RRMS among $33.3 \%$ to $89 \%[8-11,13,15,19-21,29,30]$ and chronic progressive course among $16.3 \%$ to $37.1 \%$ $[8,10-13,15,20,21,29,30]$ patients. The present study observed RRMS among $100 \%$ patients with early onset MS and 75\% patients with late onset MS. The Italian study reported RRMS among $84.5 \%$ and 79.5\% patients with early and late onset MS respectively [15].

Presence of OCBs in the CSF were found in close to three-fourth of the patients. It is reported to be present in over 95\% patients with MS [31]. It indicates an immuno-pathological process involving an abnormal synthesis of gamma globulins in the CSF. Its presence in CSF but absence in the serum reflects a local B-cell response accompanying inflammation in the central nervous system. Its presence with the carriage of HLADRB1*15 hastens disease progression [32]. Raised protein level was another common finding in CSF among patients in this study, substantiating the inflammatory role of this disease [33]. Increase in adenosine deaminase levels were seen in few patients. Therefore it needs to be included among the differential diagnosis of disorders with
ADA elevation in the CSF [34]. Collagen profile showed raised anti-nuclear antibody titre in 33.3\% of patients in this study. This is reported to increase in the early stages of MS and it later normalizes within a year [35].

The most common drug used for management in this study was steroids probably because good number of patients in this study had relapses with disabilities. In the Sudanese study, methylprednisolone and prednisolone were given among $65 \%$ and $7 \%$ MS patients respectively [21]. Steroids are preferred drugs used for managing disabling relapses such as motor system disabilities, optic neuritis and acute ataxia [36]. Steroids inhibit proinflammatory cytokines and apoptosis of $\mathrm{T}$ cells thereby, benefit patients with autoimmune conditions like MS [37]. Immunosuppressant drug, azathioprine was prescribed for $43.5 \%$ patients in this study and among $34.7 \%$ patients in the Egyptian study [8]. DMDs were given among $39.1 \%$ patients in this study and among 19.3\% [8], 37\% [16], 55.4\% [11], $72 \%$ [10] patients in other studies. In this study 50\% patients, and in the Canadian study[16] 41.2\% patients with RRMS, were managed with DMDs. Use of DMDs in the management of RRMS benefits patients by reducing both frequency and severity of relapses and helps minimize the risk of disability and nerve atrophy among them [38]. Half of the RRMS patients in this study were not treated with DMDs probably due to its high cost. Non-pharmacological methods for management constituted only physiotherapy, advised among 56.5\% patients. Other non-pharmacological methods like yoga therapy were not part of MS management. Yoga has been found to be beneficial in the management of neurogenic bladder [39].

Although non improvement with treatment were reported among $17.4 \%$ patients, no casualties were observed in this study. On the contrary, the Egyptian study reported 2(0.6\%) deaths among the MS patients [8].

A limitation of the study was that there were missing information in few case records.

\section{CONCLUSIONS}

The age at diagnosis among patients was found to be delayed at this setting. Almost three fourth of MS patients were females. Juxtacortical region was the most common site of involvement. RRMS was the most common course of the disease. The proportion of progressive cases were minimal. Steroids were the most common drug used for MS management. Non-pharmacological methods were minimally used for MS management in the setting. Even though there was no casualty, disabling symptoms like visual impairment, muscle weakness, paralysis, 
bowel and bladder impairment, and psychiatric morbidities were present among patients. These disabling morbidities might have caused impairment in the quality of life of MS patients in this setting. Hence MS need to be diagnosed early, particularly among high risk groups, like the female population and managed. In order to recognize its varied clinical presentation and course of the disease, a comprehensive clinical assessment among patients along with use of more non-pharmacological interventions is required in patient care at this setting.

\section{Acknowledgements}

The authors of this study thank the Medical Superintendents of Government Wenlock Hospital, Mangalore, Kasturba Medical College Hospital, Attavar, Mangalore and Father Muller Medical College Hospital, Mangalore for permitting us to do this study at these hospitals. We also thank Dr Misri Z K, Associate Professor, Department of Neurology, Kasturba Medical College Hospital, Ambedkar Circle, Mangalore for content validating the proforma used for data collection.

\section{Conflict of interest: none declared} Financial support: none declared

\section{REFERENCES}

1. Segal BM. Multiple Sclerosis. In: Rich RR, Shearer WT, Frew AJ, Fleisher TA, Schroeder HW, Weyand CM, editors. Clinical Immunology Principles and Practice. 5th ed. Amsterdam: Elsevier; 2019:891.

2. GBD 2016 Multiple Sclerosis Collaborators. Global, regional, and national burden of multiple sclerosis 1990-2016: a systematic analysis for the Global Burden of Disease Study 2016. Lancet Neurol. 2019;18(3):269-285.

3. Pandit L, Kundapur R. Prevalence and patterns of demyelinating central nervous system disorders in urban Mangalore, South India. Mult Scler. 2014;20(12):1651-1653.

4. Koch-Henriksen N, Sorensen PS. The changing demographic pattern of multiple sclerosis epidemiology. Lancet Neurol. 2010;9(5):520-532.

5. Derfuss T. Personalized medicine in multiple sclerosis: hope or reality? BMC Med. 2012;10:116.

6. Cross AH, Wingerchuk DM, Weinshenker BG. Active and progressive: a new duality of MS classification. Neurology. 2014;83(3):206-207.

7. Cook SD, Dhib-Jalbut S, Dowling $P$, et al. Use of magnetic resonance imaging as well as clinical disease activity in the clinical classification of multiple sclerosis and assessment of its course: A report from an international CMSC consensus conference, March 5-7, 2010. Int J MS Care. 2012;14(3):105-114.

8. Shehata HS, Shalaby NM. Demographic and clinical profile among multiple sclerosis patients in Egypt. ECTRIMS Online Library. 2015;115156:666.

9. Syal P, Prabhakar S, Thussu A, et al. Clinical profile of multiple sclerosis in north-west India. Neurol India. 1999;47(1):12-17.

10. Negreiros AA, Sousa-Munoz RL, Oliveira BE, et al. Clinical and epidemiological profile of patients diagnosed with multiple sclerosis in Joao Pessoa, Paraíba, Brazil. Arq Neuropsiquiatr. 2015;73(9):741-745.

11. Singhal A, Bhatia R. Clinical and demographic profile of multiple sclerosis patients in India: Registry database of MS patients from All India Institute of Medical Sciences (AlIMS), New Delhi. Neurology. 2014;82(10 Supplement):P5.151.

12. Gangopadhyay G, Das SK, Sarda P, et al. Clinical profile of multiple sclerosis in Bengal. Neurol India. 1999;47(1):18-21.

13. Sanaie BA, Zahwa B, Singh H. Clinical profile of multiple sclerosis in Kashmir (India): A tertiary care hospital based study. Int I Sci Stud. 2017;4(11):103-106.

14. Bigi S, Banwell B. Pediatric multiple sclerosis. J Child Neurol. 2012;27(11):1378-83.

15. Marrosu MG, Pischedda MP, Spinicci G, et al. Multiple sclerosis: Clinical profile in 71 cases. Pediatr Neurol. 1992;8(5):373.

16. Shirani A, Zhao Y, Petkau J, et al. Multiple sclerosis in older adults: the clinical profile and impact of interferon Beta treatment. Biomed Res Int. 2015;2015:451912.

17. Cornea A, Dijmarescu C, Tudor R, et al. The prevalence and clinical profile of multiple sclerosis in Timis county, Romania. Ro J Neurol. 2016;15(3):113-118.

18. Turcotte LA, Marrie RA, Patten SB, Hirdes JP. Clinical profile of persons with Multiple Sclerosis across the continuum of care. Can I Neurol Sci. 2018;45(2):188-198.

19. Santos M, Barreto LM, Lisboa Neto C, Cabeca SHL. Clinical and epidemiological profile of multiple sclerosis patients register in a reference center in para state, Amazon region, Brazil. J Neurol Sci. 2017;381:244.

20. Zahoor I, Asimi R, Haq E, Wanib IY. Demographic and clinical profile of multiple sclerosis in Kashmir: A short report. Mult Scler Relat Disord. 2017;13:103-106.

21. Ibrahim EAA, Gassoum A, Aldeaf SAH, et al. The patterns of clinical presentation of multiple sclerosis in patients admitted to the National Center of Neurological Sciences, Khartoum, Sudan 2018. J Neurol Neurosci. 2018;9(3):258.

22. Fairweather D, Frisancho-Kiss $S$, Rose NR. Sex differences in autoimmune disease from a pathological perspective. Am J Pathol. 2008;173(3):600-609.

23. Malli C, Pandit L, D'Cunha A, Mustafa S. Environmental factors related to multiple sclerosis in Indian population. PLoS One. 2015; 10(4):e0124064.

24. Pandit L, Ramagopalan SV, Malli C, et al. Association of vitamin D and multiple sclerosis in India. Mult Scler. 2013;19(12):1592-1596.

25. Kuroiwa Y, Shibashaki H, Tabira T. Clinical picture of multiple sclerosis in Asia. In: Kuroiwa Y, Kurland LT, editors. Multiple sclerosis - East and West. Fukuoka: Kyushu University Press; 1982:43-47.

26. Alroughani R, Ahmed SF, Behbahani R, et al. Increasing prevalence and incidence rates of multiple sclerosis in Kuwait. Mult Scler. 2014; 20(5):543-547.

27. Nagaraj K, Taly AB, Gupta A, et al. Depression and sleep disturbances in patients with multiple sclerosis and correlation with associated fatigue. J Neurosci Rural Pract. 2013;4(4):387-391.

28. Miller D, Barkhof F, Montalban X, et al. Clinically isolated syndromes suggestive of multiple sclerosis, part I: natural history, pathogenesis, diagnosis, and prognosis. Lancet Neurol. 2005;4(5):281-288.

29. Marzullo A, Kocevar G, Stamile C, et al. Classification of multiple sclerosis clinical profiles via graph convolutional neural networks. Front Neurosci. 2019;13:594.

30. Arewasikporn A, Ehde DM, Alschuler KN, et al. Positive factors, pain, and function in adults with multiple sclerosis. Rehabil Psychol. 2018; 63:612-620.

31. Freedman MS, Thompson EJ, Deisenhammer F, et al. Recommended standard of cerebrospinal fluid analysis in the diagnosis of multiple sclerosis: a consensus statement. Arch Neurol. 2005; 62(6):865-870.

32. Imrell K, Greiner E, Hillert J, Masterman T. HLA-DRB115 and cerebrospinal-fluid-specific oligoclonal immunoglobulin $\mathrm{G}$ bands lower age at attainment of important disease milestones in multiple sclerosis. J Neuroimmunol. 2009;210(1-2):128-130. 
33. Domingues RB, Fernandes GBP, Leite FBVM, et al. The cerebrospinal fluid in multiple sclerosis: far beyond the bands. Einstein (Sao Paulo). 2017;15(1):100-104.

34. Samuraki M, Sakai K, Odake Y, et al. Multiple sclerosis showing elevation of adenosine deaminase levels in the cerebrospinal fluid. Mult Scler Relat Disord. 2017; 13:44-46.

35. Antiguedad A, Ruiz J, Mendibe MM, Zerranz JJ. Los anticuerpos antinucleares en la esclerosis múltiple [Antinuclear antibodies in multiple sclerosis]. Neurologia. 1997; 12(6):245-248.

36. Pandit L, Murthy JM. Treatment of multiple sclerosis. Ann Indian Acad Neurol. 2011; 14(Suppl 1):S65-S69.
37. Gold R, Buttgereit F, Toyka KV. Mechanism of action of glucocorticosteroid hormones: possible implications for therapy of neuroimmunological disorders. J Neuroimmunol. 2001;117(1-2):1-8.

38. Goodin DS. Disease-modifying therapy in multiple sclerosis: update and clinical implications. Neurology. 2008; 71(24 Suppl 3):S8-13.

39. Patil NJ, Nagaratna R, Garner C, et al. Effect of integrated Yoga on neurogenic bladder dysfunction in patients with multiple sclerosis - A prospective observational case series. Complement Ther Med. 2012;20(6):424-430. 